\title{
OPEN Diagnostic performance of core needle biopsy for nodal recurrences in patients with head and neck squamous cell carcinoma
}

\author{
Ta-Hsuan Lo ${ }^{1,2}$, Cheng-Ping Wang ${ }^{1}$, Chun-Nan Chen ${ }^{1}$, Tsung-Lin Yang ${ }^{1}$, Pei-Jen Lou ${ }^{1}$, \\ Jenq-Yuh Ko ${ }^{1}$, Yih-Leong Chang ${ }^{3,4}$ \& Tseng-Cheng Chen ${ }^{1 \bowtie}$
}

This study investigated the diagnostic accuracy and affecting factors of ultrasound (US)-guided core-needle biopsy (CNB) in patients with treated head and neck squamous cell carcinoma (HNSCC). We retrospectively reviewed patients with treated HNSCC who received US-guided CNB from January 2011 to December 2018 with corresponding imaging. Pathological necrosis and fibrosis of targeted lymph nodes (LNs) were evaluated. We analyzed the correlation between CNB accuracy and clinical and pathological characteristics. In total, 260 patients were included. The overall sensitivity, specificity, positive predictive value (PPV), negative predictive value (NPV), and accuracy of CNB for nodal recurrence were $84.47 \%, 100 \%, 100 \%, 54.67 \%$, and $86.92 \%$, respectively. CNB of fibrotic LNs had significantly worse sensitivity, NPV, and accuracy than that of non-fibrotic LNs. Similarly, CNB of necrotic LNs had significantly worse sensitivity, NPV, and accuracy than non-necrotic LNs. Multivariate regression revealed that fibrotic $L N$ was the only independent factor for a true positive rate, whereas both necrotic $L N$ and fibrotic $L N$ were independent factors for a false negative rate. The diagnostic accuracy of CNB in treated HNSCC patients is affected by LN necrosis and fibrosis. Therefore, CNB results, particularly for necrotic or fibrotic LNs, should be interpreted carefully.

In patients with treated head and neck squamous cell carcinoma (HNSCC), detection of neck nodal recurrence is an important issue. Therefore, imaging techniques like high-resolution ultrasound (US) ${ }^{1}$, computed tomography $(\mathrm{CT})$, magnetic resonance imaging (MRI) and positron-emission tomography (PET) ${ }^{2-6}$ play important roles in the evaluation of suspected neck nodal lesions during follow up. However, imaging studies are only able to provide indirect evidence of the lesion but not direct cytological or pathological evidence to help clinicians differentiate malignant lesions from other conditions affecting the neck, such as deep neck infection, necrosis, or chyle/hematoma accumulation. Therefore, obtaining tissue samples remains the standard requirement for accurate diagnosis of neck nodal recurrence.

In treated HNSCC patients, open biopsy of the neck provides a tissue sample that is ideal for immunohistochemical analysis. However, this procedure usually requires general anesthesia and hospital admission. Previous reports have shown that prior radiation and/or neck dissection can result in significant changes to the histological architecture of the neck, for example, tissue fibrosis or necrosis ${ }^{7,8}$. Therefore, an open surgical biopsy without guidance for the suspicious lesion over treated neck is always difficult and carries risk of injury to the important vessels or nerves. Recently, US-guided lymph node (LN) core-needle biopsy (CNB) has been shown to be as advantageous as open surgical biopsies but without the disadvantages associated with open surgical biopsies ${ }^{9,10}$. It is minimally invasive, safe, simple, and cheap. In addition, it is also a fast outpatient procedure that can be performed under local anesthesia and provides an acceptable cutting tissue core for immunohistochemical study. However, to the best of our knowledge, there have been no comprehensive studies investigating whether changes to the histological architecture in treated neck have any impact on the diagnostic accuracy of cutting tissue core by US-guided CNB. Therefore, in this study, we aim to study the performance of US-guided CNB in treated

\footnotetext{
${ }^{1}$ Department of Otolaryngology, National Taiwan University Hospital and National Taiwan University College of Medicine, No.7, Chung-Shan S. Rd., Taipei 10002, Taiwan, ROC. ${ }^{2}$ Department of Otolaryngology, National Taiwan University Hospital Hsin-Chu Biomedical Park Branch, Hsinchu, Taiwan, ROC. ${ }^{3}$ Department of Pathology, National Taiwan University Hospital, National Taiwan University Cancer Center and National Taiwan University College of Medicine, Taipei, Taiwan, ROC. ${ }^{4}$ Graduate Institute of Pathology, National Taiwan University College of Medicine, Taipei, Taiwan, ROC. ${ }^{\square}$ email: tcchen0102@ntu.edu.tw
} 


\begin{tabular}{|c|c|c|c|}
\hline Characteristics & Non-recurrent LN $(\mathrm{N}=41)$ & Recurrent LN (N=219) & $p$ value \\
\hline \multicolumn{4}{|l|}{ Age (years) } \\
\hline$\geqq 50$ & $29(70.73 \%)$ & $173(79 \%)$ & \multirow{2}{*}{0.31} \\
\hline$<50$ & $12(29.27 \%)$ & $46(21 \%)$ & \\
\hline \multicolumn{4}{|l|}{ Gender } \\
\hline Male & $38(92.68 \%)$ & $198(90.41 \%)$ & \multirow{2}{*}{$0.78^{*}$} \\
\hline Female & $3(7.32 \%)$ & $21(9.59 \%)$ & \\
\hline \multicolumn{4}{|l|}{ Primary index tumor } \\
\hline Oral cancer & $15(36.59 \%)$ & $121(55.25 \%)$ & \multirow{5}{*}{$0.02^{*}$} \\
\hline p16- $(<70 \%)$ Oropharyngeal cancer & $12(29.27 \%)$ & $44(20.09 \%)$ & \\
\hline p16+( $\geqq 70 \%)$ Oropharyngeal cancer & $2(4.88 \%)$ & $1(0.46 \%)$ & \\
\hline Hypopharyngeal, Laryngeal cancer & $10(24.39 \%)$ & $31(14.16 \%)$ & \\
\hline Others & $2(4.88 \%)$ & $22(10.05 \%)$ & \\
\hline \multicolumn{4}{|l|}{ Previous neck management } \\
\hline Neck dissection only & $9(21.95 \%)$ & $64(29.22 \%)$ & \multirow{4}{*}{0.20} \\
\hline Radiation only & $22(53.66 \%)$ & $47(21.46 \%)$ & \\
\hline Neck dissection and Radiation & $10(24.39 \%)$ & $103(47.03 \%)$ & \\
\hline No treatment & 0 & $5(2.28 \%)$ & \\
\hline \multicolumn{4}{|l|}{ Neck recurrence duration } \\
\hline Acute ( $\leqq 6$ months) & $16(39.02 \%)$ & $66(30.14 \%)$ & \multirow{2}{*}{0.28} \\
\hline Non-Acute (> 6 months) & $25(60.98 \%)$ & $153(69.86 \%)$ & \\
\hline \multicolumn{4}{|l|}{ Target $\mathrm{LN}$ pathological fibrosis } \\
\hline Fibrotic changes+ & $17(41.46 \%)$ & $55(25.11 \%)$ & \multirow{2}{*}{0.04} \\
\hline Fibrotic changes- & $24(58.54 \%)$ & $164(74.89 \%)$ & \\
\hline \multicolumn{4}{|l|}{ Target LN pathological necrosis } \\
\hline Necrosis+ & $8(19.51 \%)$ & $34(15.53 \%)$ & \multirow{2}{*}{0.64} \\
\hline Necrosis- & $33(80.49 \%)$ & $185(84.47 \%)$ & \\
\hline \multicolumn{4}{|l|}{ Target LN radiological necrosis } \\
\hline Total necrosis & $2(4.88 \%)$ & $26(11.87 \%)$ & \multirow{3}{*}{$0.29^{*}$} \\
\hline Partial necrosis & $9(21.95 \%)$ & $59(26.94 \%)$ & \\
\hline No necrosis & $30(73.17 \%)$ & $134(61.19 \%)$ & \\
\hline \multicolumn{4}{|l|}{ Targeted LN level in neck } \\
\hline Upper (I/II/Va) & $25(60.98 \%)$ & $150(68.49 \%)$ & \multirow{2}{*}{0.37} \\
\hline Lower (III/IV/Vb) & $16(39.02 \%)$ & $69(31.51 \%)$ & \\
\hline \multicolumn{4}{|l|}{ LN width size } \\
\hline$\geqq 1.5 \mathrm{~cm}$ & $21(51.23 \%)$ & $179(81.74 \%)$ & \multirow{2}{*}{$<0.001$} \\
\hline$<1.5 \mathrm{~cm}$ & $20(48.78 \%)$ & $40(18.26 \%)$ & \\
\hline \multicolumn{4}{|l|}{ Ultrasound characteristic } \\
\hline Ill-defined & $21(51.22 \%)$ & $83(37.90 \%)$ & 0.12 \\
\hline Heterogenous & $20(48.78 \%)$ & $89(40.64 \%)$ & 0.39 \\
\hline \multicolumn{4}{|l|}{ CNB tissue diagnosis } \\
\hline Positive & 0 & $185(84.47 \%)$ & \multirow{2}{*}{$<0.001$} \\
\hline Negative & $41(100 \%)$ & $34(15.53 \%)$ & \\
\hline
\end{tabular}

Table 1. Basic, clinicopathologic and ultrasound characteristics of treated head and neck SCC patients with recurrent LN. SCC, squamous cell carcinoma; LN, lymph node. ${ }^{\star}$ Using Fisher's exact test.

HNSCC patients. All possible factors, especially the fibrosis and necrosis in targeted LN, will be examined in order to clarify their impact, if any, on the performance of US-guided CNB in detail.

\section{Results}

Patient demographics. In our study, a total of 260 patients with treated HNSCC who had a suspected neck mass received US-guided CNB were included. Of the 260 patients, 236 (90.77\%) were men and 24 (9.23\%) were women. Their ages ranged from 30 to 88 years, with a mean age of $57 \pm 10$ years. The long axis of targeted LN ranged from 0.6 to $5.20 \mathrm{~cm}$, with a mean axis of $2.2 \pm 0.8 \mathrm{~cm}$. Totally, there were $41(15.77 \%)$ non-recurrent patients and $219(84.23 \%)$ recurrent patients, including 185 patients confirmed by US-guided CNB and 34 patients confirmed by progressive disease or salvage neck dissection, in our series. The basic clinicopathologic and US characteristics of these recurrent and non-recurrent patients are shown in Table 1. There were significant 
differences between the patients with non-recurrent and recurrent $\mathrm{LN}$ in terms of primary tumor sites $(p=0.02)$, target LN pathological fibrosis $(p=0.04)$ and LN width size $(p<0.001)$.

The association between the presence of necrotic/fibrotic LNs and other clinical/pathological characteristics. In our study, there were $72(27.69 \%)$ patients with fibrotic LNs pathologically and 123 (47.31\%) patients with necrotic LNs (81 patients with necrotic LNs radiologically, 27 patients with necrotic LNs pathologically, and 15 patients with combined radiologically and pathologically necrotic LNs). Among the 42 patients with necrotic LN pathologically, there were 8 patients with tissue necrosis and 34 patients with tumor necrosis. We attempted to clarify the association between the presence of necrotic/fibrotic LNs and underlying clinical/pathological characteristics of patients with treated HNSCC. Compared with 58 patients aged $<50$ years, the odds ratio (OR)s of necrotic/fibrotic LNs in 202 patients aged $\geq 50$ years were not significantly different ( $p=0.47$ and 0.72 , respectively). Compared to 24 women, the ORs of necrotic/fibrotic LNs in 236 men were not significantly different ( $p=0.48$ and 0.76 , respectively). Compared to 201 patients with treated non-oropharyngeal cancer, the ORs of necrotic/fibrotic LNs in 59 patients with treated oropharyngeal cancer were not significantly different ( $p=0.54$ and 0.44 , respectively). Compared to 191 patients without prior radiation, the ORs of necrotic LNs in 69 patients with prior radiation was not significantly different $(p=0.31)$. However, the OR of fibrotic LNs was significantly high for the patients with prior radiation (OR $1.91,95 \%$ confidence interval $1.06-3.44, p=0.03)$. Compared to 178 patients with recurrent duration $>6$ months, the ORs of necrotic LNs in 82 patients with recurrent duration $\leq 6$ months was not significantly different $(p=0.74)$. However, the OR of fibrotic LNs was significantly high for the patients with recurrent duration $\leq 6$ months (OR 1.93, 95\% confidence interval 1.10-3.39, $p=0.02$ ) Compared to 85 patients with lower neck LN, the ORs of necrotic/fibrotic LNs in 175 patients with upper neck LN were not significantly different ( $p=0.84$ and 0.47 , respectively). Finally, compared to 60 patients with LN width size $<1.5 \mathrm{~cm}$, the ORs of necrotic/fibrotic LN in 200 patient with LN width size $\geqq 1.5 \mathrm{~cm}$ were not significantly different ( $p=0.88$ and 0.86 , respectively).

The sensitivity, specificity, PPV, NPV and accuracy of US-guided CNB tissue sampling method in necrotic and fibrotic LN. The overall sensitivity, specificity, PPV, NPV, and accuracy of US-guided CNB in our study were $84.47 \%, 100 \%, 100 \%, 54.67 \%$, and $86.92 \%$, respectively (Fig. 1A). According to the pathological results from $\mathrm{CNB}$, none of the patients had non-diagnostic findings. Compare to the targeted LN without pathological fibrosis, the targeted LN with pathological fibrosis had a significantly worse sensitivity $(p<0.00001)$, NPV $(p<0.003)$ and accuracy $(p<0.00001$, Fig. 1B). Similarly, compared to the targeted LN without radiologi$\mathrm{cal} /$ pathological necrosis, the targeted LN with radiological/pathological necrosis had a significantly worse sensitivity $(p<0.00001)$, NPV $(p<0.00001)$, and accuracy $(p<0.00001$, Fig. $1 C)$.

Finally, regarding the true positive rates and false negative rates, all of the characteristics between recurrent and non-recurrent patients were further examined by multivariate logistic regression analysis. This analysis revealed that fibrotic LNs (relative risk (RR) $0.21,95 \%$ CI $0.11-0.40, p<0.001$, Fig. $2 \mathrm{~A}$ ) was the only independent risk factor for decreased true positive rates. Regarding false negative rates, necrotic LNs (RR 2.66, 95\% CI $1.03-6.84, p=0.04$ ) and fibrotic LNs (RR 6.18, 95\% CI 2.77-13.82, $p<0.001$ ) were independent risk factors for increased false negative rates (Fig. 2B). To summarize, the necrotic LNs and fibrotic LNs had significant impacts on true positive and false negative rates of US-guided CNB.

\section{Discussion}

Previously, US-guided CNB has been reported as an effective, inexpensive, time-efficient, safe, and minimally invasive diagnostic tool for neck nodal disease $\mathrm{s}^{11,12}$. According to previous reports, the sensitivity, specificity, and accuracy of CNB for neck masses were about $99.7 \%, 100 \%$ and $99.46 \%$, respectively ${ }^{13,14}$. However, data on the diagnostic power of CNB came majorly from neck masses without obvious histological or morphological changes. To the best of our knowledge, no previous work has focused on the possible impact of morphological and histological changes in neck masses on the diagnostic power of CNB. According to our study, several important findings should be emphasized. First, patients with prior neck radiation and recurrent duration $\leqq 6$ months had significantly high incidence of fibrotic LNs.. Second, necrotic and fibrotic LNs had significant impact on the diagnostic performance of CNB, especially for the true positive and false negative rates.

Compared to open surgical biopsy, US-guided CNB had significantly lower sequela and therefore is more accepted by patients ${ }^{15}$, and could be the first choice to obtain tissue samples. However, for patients with HNSCC, it has been reported that the incidence of neck nodal necrosis ranged from 20 to 38\%, especially for HPV-related oropharyngeal cancer ${ }^{16,17}$. According to the results of our study, if there is necrotic changes in the target neck mass, the interpretation of CNB findings should be done with caution. In fact, the cutting method used during CNB may only provide partially crushed tissue (Fig. 3) or even no tissue. Previously, it had been reported that nodal necrosis could hinder the accuracy of CNB diagnosis in patients with treated $\mathrm{HNSCC}^{18}$. In our study, no clinical characteristics had a significant association with the presence of necrotic lymph nodes in treated HNSCC patients. In our opinion, it should be very important to determine the presence or absence of necrosis in the targeted LN before CNB, especially in patients with treated HNSCC. For LNs with necrotic changes, the clinician should keep in mind that the significantly low NPV of CNB can sometimes cause a dilemma for the diagnosis. In such a situation, excisional biopsy or US-guided FNA should be considered as an alternative diagnostic tool. It had been reported that US-guided FNA had good accuracy in post-radiotherapy pateints ${ }^{19}$. Besides, other functional imaging such as PET may be considered to confirm the negative findings from the CNB.

In our study, in addition to nodal necrosis, pathological fibrosis of the LNs could also hinder the diagnostic accuracy of CNB. Previous neck management, especially radiotherapy, cause an increased production of fibrin. This radiation fibrosis can affect any tissue in the radiation field, including the lymph node ${ }^{20}$. It has been reported 

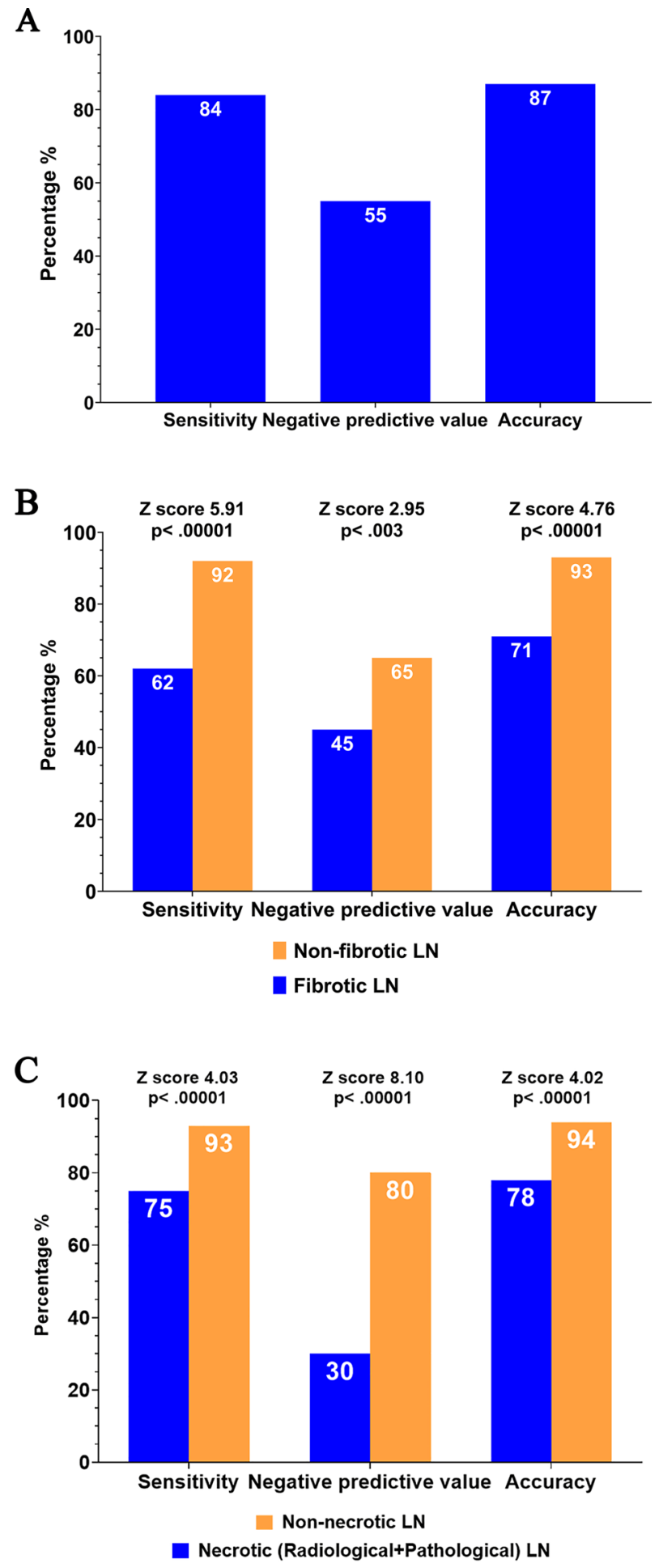

Figure 1. The sensitivity, negative predictive value, and accuracy of (A) core-needle biopsy $(\mathrm{CNB})$ in patients with treated head and neck squamous cell carcinoma, overall; (B) CNB for the lymph node (LN) with and without fibrotic LN; and (C) CNB for the LN with and without necrotic LN.

that the incidence of neck fibrosis after neck radiation can reach $22 \%{ }^{21}$. According to our results, $27 \%$ of core tissues from treated LN showed some degree of fibrotic change (Fig. 4). Furthermore, imaging methods such as MRI, CT, or US could not identify the degree of LN fibrotic change before the CNB procedure. Therefore, detection of pathological fibrotic changes majorly depended on the microscopic findings of core tissue obtained from CNB. According to our results, these fibrotic changes significantly hinder the diagnostic accuracy of CNB, 
A

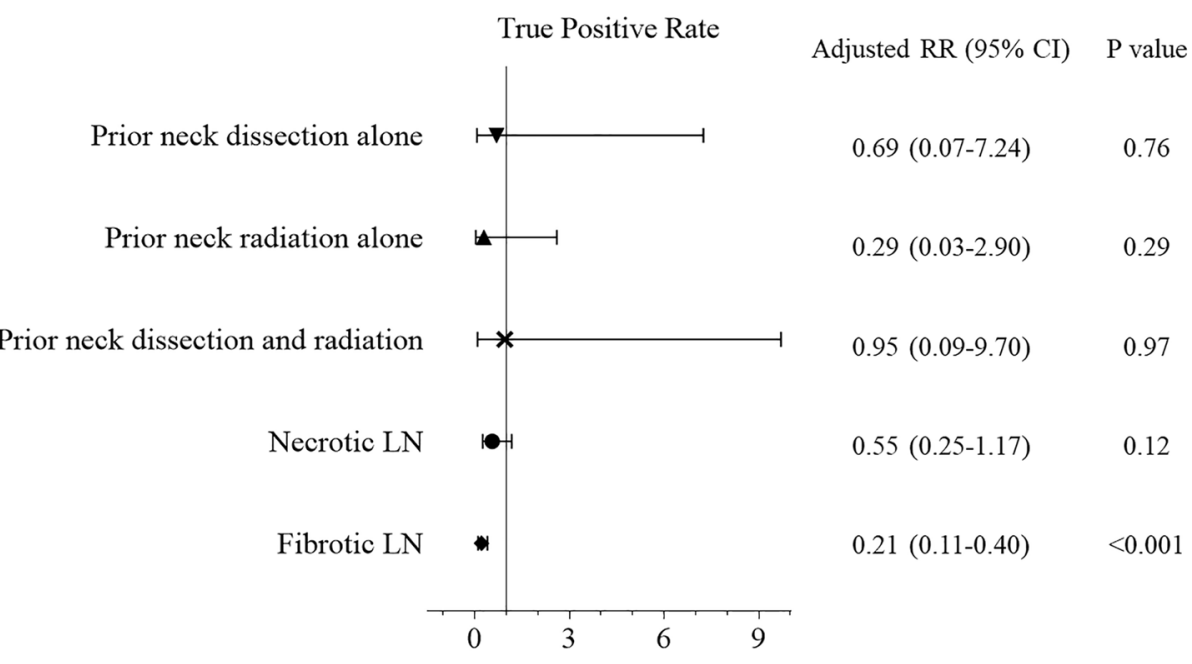

B

False Negative Rate

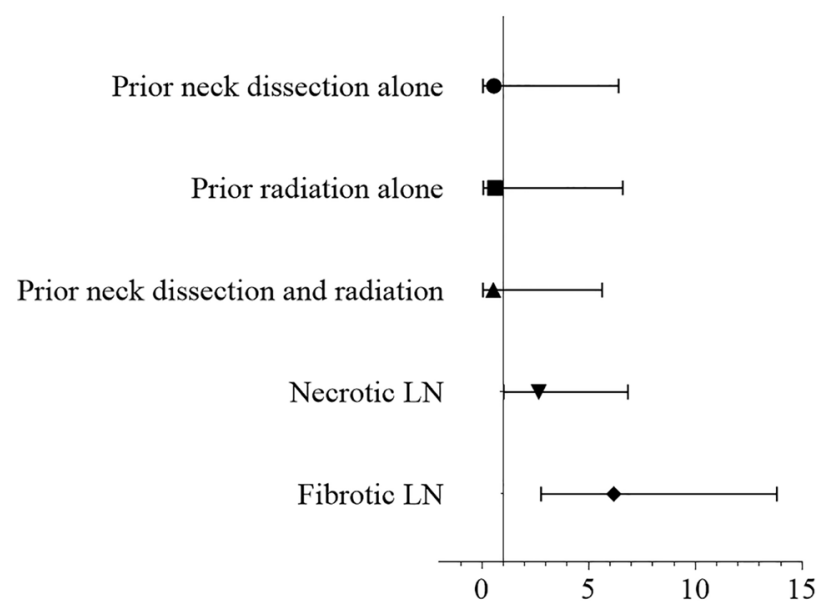

Adjusted RR (95\% CI) P value

$0.56(0.05-6.40) \quad 0.64$

$0.61(0.06-6.60) \quad 0.68$

$0.53(0.05-5.63) \quad 0.6$

$2.66(1.03-6.84) \quad 0.04$

$6.18(2.77-13.82) \quad<0.001$

Figure 2. Forest plot of multivariate analysis of possible impacting factors using the logistic regression method in (A) true positive rates and (B) false negative rates.

especially true positive and false negative rates. Also, the linear one shot procedure of CNB could potentially result in less extensive sampling.

In summary, US-guided CNB still could be the procedure of choice for treated HNSCC patients with a suspected neck mass. However, US-guided CNB could not replace excisional biopsy as the gold standard because its diagnostic accuracy can be significantly affected by necrotic and/or fibrotic LNs. The CNB pathological result should be interpreted with caution if necrosis and/or pathological fibrosis present. If the suspicion of nodal recurrence is high but the findings from $\mathrm{CNB}$ are negative, an open biopsy or other functional imaging such as PET should be considered.

There were a few limitations to our study. First, this retrospective study might include various types of bias. Even though multivariant analysis was used to evaluate the factors impacting true positive and false negative rates, there were unavoidable or unnoticed selection biases. Second, in all of the cases, the needle size (18-gauge) used during the $\mathrm{CNB}$ procedure was small. Therefore, the effect of different needle size, especially a larger size needle (16-gauge), could not be examined in our study. Third, the number of CNB passes was not available. Therefore, the diagnostic power of CNB may be under- or over-estimated. The strength of this study is that we comprehensively analyzed the association between necrotic/fibrotic changes of the core tissue and the diagnostic accuracy of CNB for treated HNSCC patients. In our opinion, a well-designed prospective randomized trial is warranted in the future to examine the diagnostic power of CNB in patients with treated HNSCC. 
A

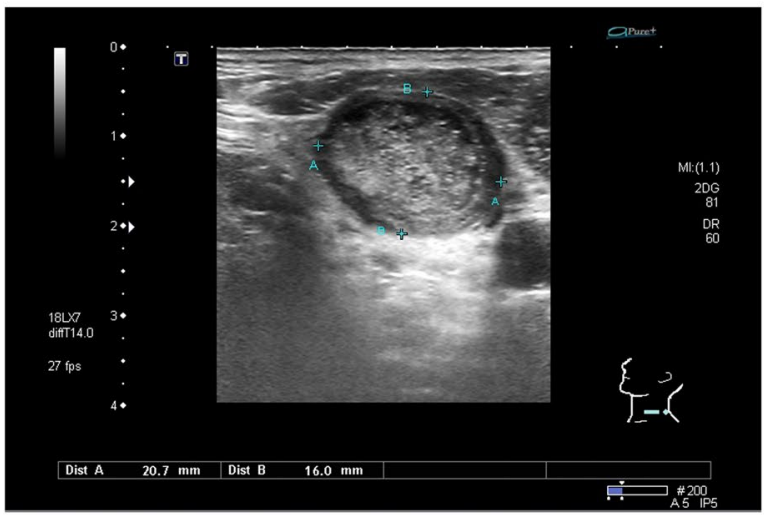

$\mathrm{C}$

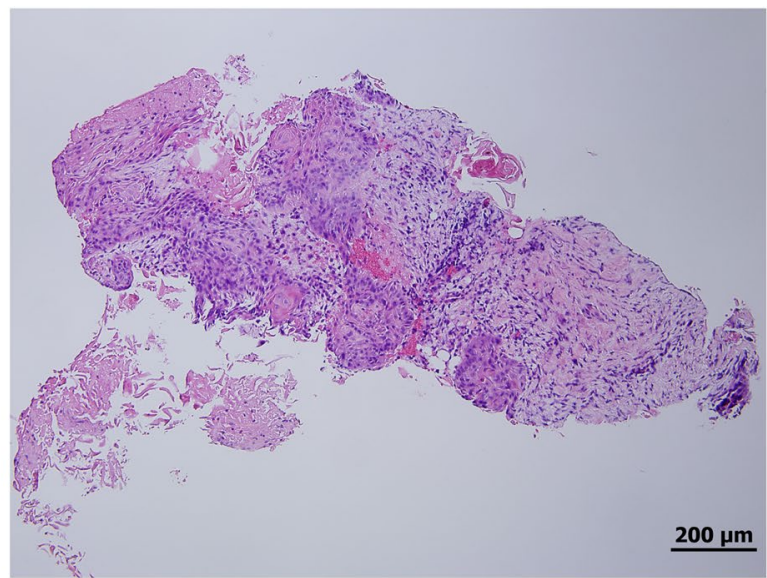

B

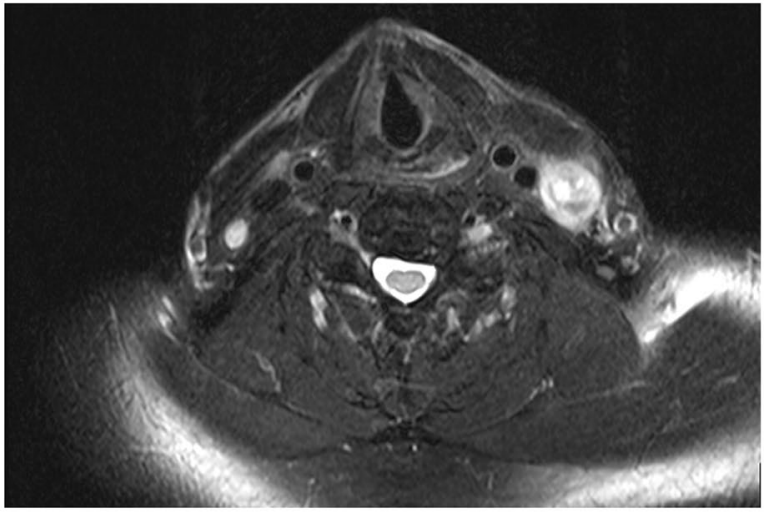

$\mathrm{D}$

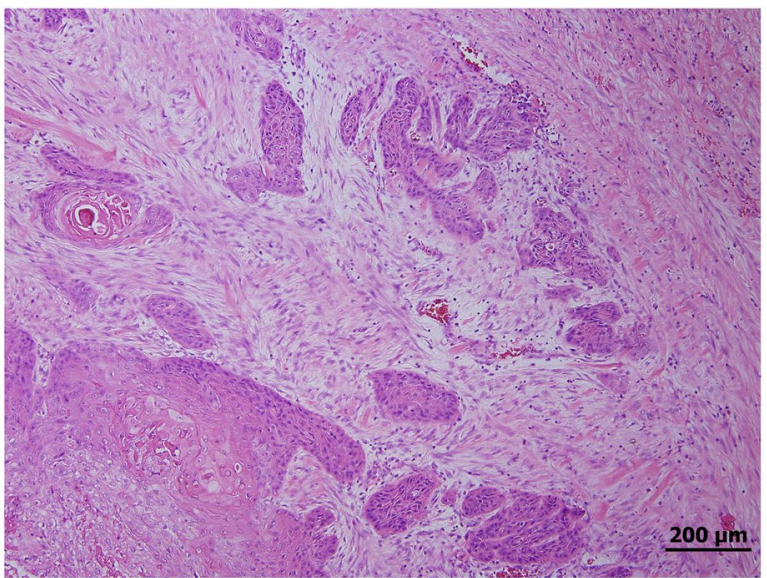

Figure 3. (A) Ultrasound image of the treated left tongue cancer with an ipsilateral level IV nodal mass; (B) corresponding magnetic resonance imaging showing necrotic changes with a T2 axial view; (C) core-needle biopsy (CNB) revealed necrotic tissue with metastatic squamous cell carcinoma (SCC) in nests infiltrated in the fibrotic stroma; and (D) tissue from salvage neck dissection revealed metastatic SCC without extranodal extension.

\section{Materials and methods}

Ethical Considerations. The study was approved by the hospital's Research Ethics Committee (NTUH IRB-201812004RINC) and all methods were performed in accordance with the relevant guidelines and regulations.

Patient population. We retrospectively reviewed the medical records of neck US from January 2011 to December 2018 at National Taiwan University Hospital. First, all patients with a definite diagnosis of HNSCC were initially included in the study. Further, patients without the history of primary curative treatment before US examination, without US-guided CNB, without corresponding cross-section imaging such as MRI and/or CT at the time point of US examination and without subsequent follow up in our hospital after US examination were excluded from our series. Therefore, all patients included in our series were treated HNSCC patients who underwent neck US-guided CNB with corresponding cross-sectional imaging (MRI or CT) and histological tissue diagnosis by US-guided CNB. Necrotic changes in the aspirated neck mass were determined by corresponding MRI and/or CT imaging before US-guided CNB and were radiologically defined as a central area of low attenuation surrounded by an irregular rim of enhancing tissue ${ }^{22}$. The size of the aspirated neck mass was determined by the long-axis on US measurement. Fibrotic changes in the aspirated neck mass were determined on the basis of the pathologic findings of US-guided CNB tissue block. In our study, all patients in our series received follow-up again 6 months later by routine survey using neck palpation, imaging CT, or MRI to exclude the misdiagnoses of US-guided CNB. The definite diagnoses of LN recurrence were based on the CNB pathologic report or progressive LN during follow up. Furthermore, the TNM stage of HNSCC was determined according to the 2010 criteria of the American Joint Committee on Cancer $^{23}$.

Procedures of US examination and US-guided CNB. Head and neck US was performed (Toshiba Aplio SSA790 diagnostic US system, Tochigi-ken, Japan or Hitachi HI VISION Avius ${ }^{\circledR}$, Soto-kanda, Chiyoda-ku, Tokyo, Japan) with a 12-MHz linear array transducer. After obtaining informed consent from the patients, US- 
A

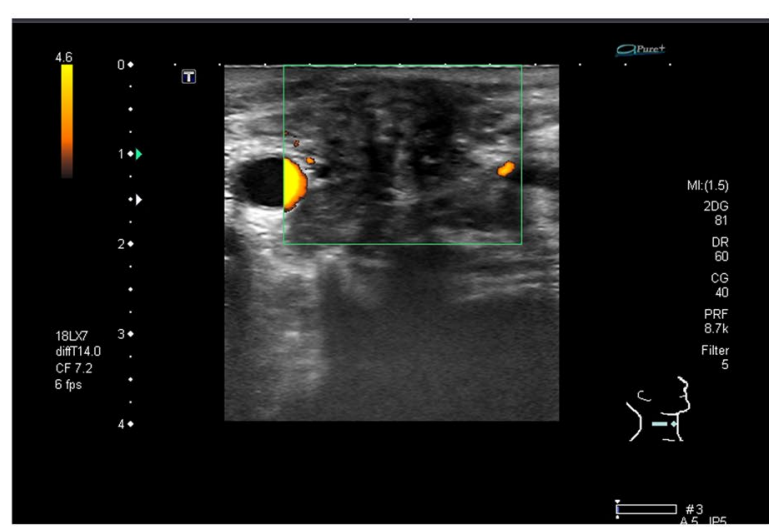

C

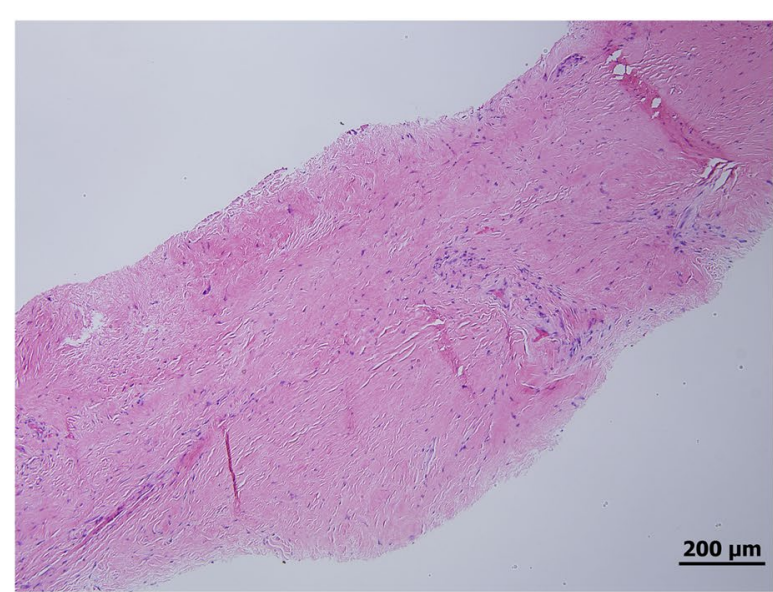

B

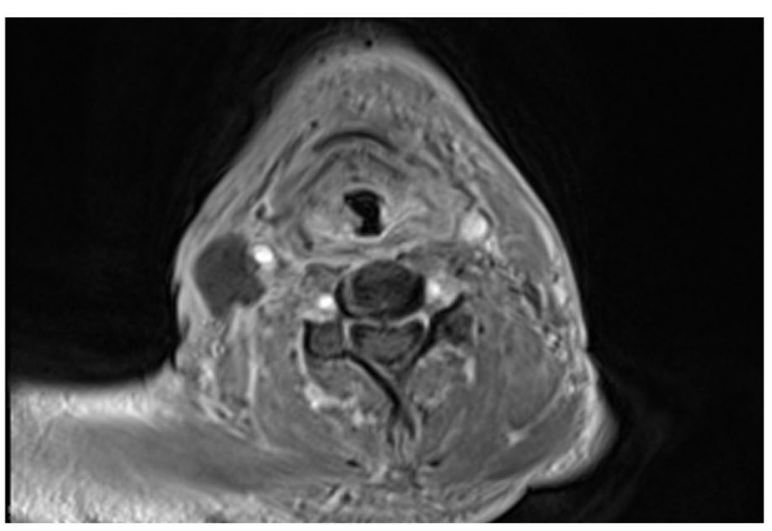

D

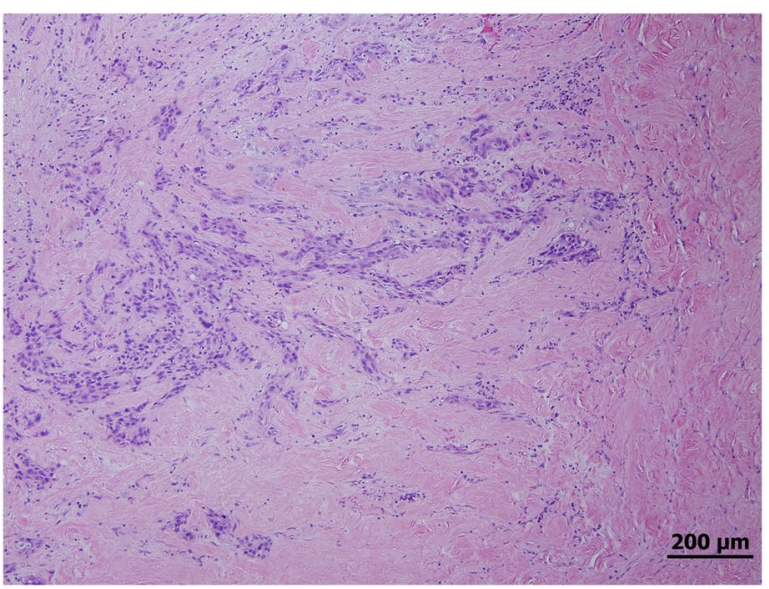

Figure 4. (A) Ultrasound picture of treated right tongue cancer with ipsilateral nodal mass; (B) corresponding magnetic resonance imaging showing axial view of T1 with gadolinium contrast medium; $(\mathbf{C})$ core-needle biopsy revealed fibrotic tissue only; (D) Open biopsy revealed fibrotic tissue with metastatic squamous cell carcinoma and focal extracellular keratin deposition.

guided CNB was carried out. CNB was performed using the free-hand technique with an 18-gauge core-needle (Temno Evolution $^{\text {tw }}$ Biopsy Devices, Cardinal Health Inc., Dublin, CA, USA) under local anesthesia, as previously described ${ }^{1}$.

Statistical analysis. All statistical analyses were performed using the SPSS software package, version 23.0 (SPSS Inc., Chicago, IL, USA). Fisher's exact tests and Chi-square tests were used to determine differences in the clinical and US features between patients with and those without necrotic LNs. The two-proportion z-test was used to compare the sensitivity, negative predictive value (NPV) and accuracy between the targeted LNs with and without histological fibrosis or radiological necrosis. The primary outcomes were the sensitivity, specificity, positive predictive value (PPV), NPV and accuracy of US-guided CNB to confirm neck nodal recurrence in treated HNSCC patients. The secondary outcomes were difference in sensitivity, NPV and accuracy of USguided CNB between the targeted LNs with and without histological fibrosis or morphological necrosis. All potential US features were further analyzed using a multivariate logistic regression model. Corresponding $p$ values $<0.05$ were interpreted as being statistically significant.

\section{Conclusion}

The diagnostic accuracy of CNB in treated HNSCC patients is affected by LN necrosis or fibrosis. Therefore, CNB results, particularly for necrotic/fibrotic LNs, should be interpreted carefully. If the suspicion of nodal recurrence is high but the findings from $\mathrm{CNB}$ are negative, an open biopsy or other functional imaging such as PET should be considered, if feasible.

Received: 26 September 2021; Accepted: 24 January 2022

Published online: 07 February 2022

\section{References}

1. Lin, C. M. et al. The application of ultrasound in detecting lymph nodal recurrence in the treated neck of head and neck cancer patients. Sci. Rep. 7, 3958. https://doi.org/10.1038/s41598-017-04039-3 (2017). 
2. Rivelli, V. et al. Screening recurrence and lymph node metastases in head and neck cancer: The role of computer tomography in follow-up. Head Neck Oncol. 3, 18. https://doi.org/10.1186/1758-3284-3-18 (2011).

3. Zhao, X. \& Rao, S. Surveillance imaging following treatment of head and neck cancer. Semin. Oncol. 44, 323-329. https://doi.org/ 10.1053/j.seminoncol.2018.01.010 (2017).

4. Payne, K. F., Haq, J., Brown, J. \& Connor, S. The role of diffusion-weighted magnetic resonance imaging in the diagnosis, lymph node staging and assessment of treatment response of head and neck cancer. Int. J. Oral Maxillofac. Surg. 44, 1-7. https://doi.org/ 10.1016/j.ijom.2014.09.008 (2015).

5. Anzai, Y. et al. Recurrence of head and neck cancer after surgery or irradiation: Prospective comparison of 2-deoxy-2-[F-18] fluoroD-glucose PET and MR imaging diagnoses. Radiology 200, 135-141. https://doi.org/10.1148/radiology.200.1.8657901 (1996).

6. de Bree, R. et al. Detection of locoregional recurrent head and neck cancer after (chemo)radiotherapy using modern imaging. Oral Oncol 45, 386-393. https://doi.org/10.1016/j.oraloncology.2008.10.015 (2009).

7. Cooper, J. S., Fu, K., Marks, J. \& Silverman, S. Late effects of radiation therapy in the head and neck region. Int. J. Radiat. Oncol. Biol. Phys. 31, 1141-1164. https://doi.org/10.1016/0360-3016(94)00421-G (1995).

8. Lukens, J. N. et al. Late consequential surgical bed soft tissue necrosis in advanced oropharyngeal squamous cell carcinomas treated with transoral robotic surgery and postoperative radiation therapy. Int. J. Radiat. Oncol. Biol. Phys. 89, 981-988. https://doi.org/ 10.1016/j.ijrobp.2014.04.024 (2014).

9. Borhani, A. A. \& Monaco, S. E. Chapter 7 Image-guided fine-needle aspiration and core needle biopsy of neck lymph nodes: Techniques, pearls, and pitfalls. Semin. Ultrasound CT MR 38, 531-541. https://doi.org/10.1053/j.sult.2017.05.007 (2017).

10. Saha, S. et al. Ultrasound guided Core Biopsy, Fine Needle Aspiration Cytology and Surgical Excision Biopsy in the diagnosis of metastatic squamous cell carcinoma in the head and neck: An eleven year experience. Eur. J. Radiol. 80, 792-795. https://doi.org/ 10.1016/j.ejrad.2010.10.020 (2011).

11. Nyquist, G. G., Tom, W. D. \& Mui, S. Automatic core needle biopsy: a diagnostic option for head and neck masses. Arch. Otolaryngol. Head Neck Surg. 134, 184-189. https://doi.org/10.1001/archoto.2007.39 (2008).

12. Akkina, S. R., Kim, R. Y., Stucken, C. L., Pynnonen, M. A. \& Bradford, C. R. The current practice of open neck mass biopsy in the diagnosis of head and neck cancer: A retrospective cohort study. Laryngoscope Investig. Otolaryngol. 4, 57-61. https://doi.org/10. 1002/lio2.240 (2019).

13. Oh, K. H. et al. Efficacy of ultrasound-guided core needle gun biopsy in diagnosing cervical lymphadenopathy. Eur. Ann. Otorhinolaryngol. Head Neck. Dis. 133, 401-404. https://doi.org/10.1016/j.anorl.2016.01.013 (2016).

14. Screaton, N. J., Berman, L. H. \& Grant, J. W. Head and neck lymphadenopathy: evaluation with US-guided cutting-needle biopsy. Radiology 224, 75-81. https://doi.org/10.1148/radiol.2241010602 (2002).

15. Jeong, E. J. et al. A comparison of ultrasound-guided fine needle aspiration versus core needle biopsy for thyroid nodules: Pain, tolerability, and complications. Endocrinol. Metab. (Seoul) 33, 114-120. https://doi.org/10.3803/EnM.2018.33.1.114 (2018).

16. Goldenberg, D. et al. Cystic lymph node metastasis in patients with head and neck cancer: An HPV-associated phenomenon. Head Neck 30, 898-903. https://doi.org/10.1002/hed.20796 (2008).

17. Cantrell, S. C. et al. Differences in imaging characteristics of HPV-positive and HPV-Negative oropharyngeal cancers: a blinded matched-pair analysis. AJNR Am. J. Neuroradiol. 34, 2005-2009. https://doi.org/10.3174/ajnr.A3524 (2013).

18. Wagner, J. M. et al. Ultrasound-guided fine-needle aspiration with optional core needle biopsy of head and neck lymph nodes and masses: comparison of diagnostic performance in treated squamous cell cancer versus all other lesions. J. Ultrasound Med. 38, 2275-2284. https://doi.org/10.1002/jum.14918 (2019).

19. Lo, W. C. et al. The effect of radiotherapy on ultrasound-guided fine needle aspiration biopsy and the ultrasound characteristics of neck lymph nodes in oral cancer patients after primary treatment. PLoS ONE 11, e0149346. https://doi.org/10.1371/journal. pone.0149346 (2016).

20. Shvero, J. et al. Histological changes in the cervical lymph nodes after radiotherapy. Oncol Rep 8, 909-911. https://doi.org/10.3892/ or.8.4.909 (2001)

21. Hamilton, S. N. et al. Documentation and incidence of late effects and screening recommendations for adolescent and young adult head and neck cancer survivors treated with radiotherapy. Support Care Cancer 27, 2609-2616. https://doi.org/10.1007/s00520018-4559-5 (2019).

22. Chong, V. F., Fan, Y. F. \& Khoo, J. B. MRI features of cervical nodal necrosis in metastatic disease. Clin. Radiol. 51, 103-109. https:// doi.org/10.1016/s0009-9260(96)80265-0 (1996).

23. Edge, S. B. \& Compton, C. C. The American Joint Committee on Cancer: the 7th edition of the AJCC cancer staging manual and the future of TNM. Ann. Surg. Oncol. 17, 1471-1474. https://doi.org/10.1245/s10434-010-0985-4 (2010).

\section{Acknowledgements}

We thank the staff of the Eighth Core Lab, Department of Medical Research for technical support during the study. We thank the Ministry of Science and Technology, Taiwan (109-2314-B-002-054) and National Taiwan University Hospital, Taiwan (108-004365) for grant support during the study.

\section{Author contributions}

T.H.L. and T.C.C. analyzed the data and wrote the manuscript; C.P.W., C.N.C., T.L.Y., P.J.L., J.Y.K., and Y.L.C. designed and performed the project. All authors discussed the results and implications and commented on the manuscript at all stages.

\section{Funding}

We thank the Ministry of Science and Technology, Taiwan (109-2314-B-002-054) and National Taiwan University Hospital, Taiwan (108-004365) for grant support during the study.

\section{Competing interests}

The authors declare no competing interests.

\section{Additional information}

Correspondence and requests for materials should be addressed to T.-C.C.

Reprints and permissions information is available at www.nature.com/reprints.

Publisher's note Springer Nature remains neutral with regard to jurisdictional claims in published maps and institutional affiliations. 
(c) (i) Open Access This article is licensed under a Creative Commons Attribution 4.0 International cc) License, which permits use, sharing, adaptation, distribution and reproduction in any medium or format, as long as you give appropriate credit to the original author(s) and the source, provide a link to the Creative Commons licence, and indicate if changes were made. The images or other third party material in this article are included in the article's Creative Commons licence, unless indicated otherwise in a credit line to the material. If material is not included in the article's Creative Commons licence and your intended use is not permitted by statutory regulation or exceeds the permitted use, you will need to obtain permission directly from the copyright holder. To view a copy of this licence, visit http://creativecommons.org/licenses/by/4.0/.

(C) The Author(s) 2022 